\title{
KCC2 expression levels are reduced in post mortem brain tissue of Rett syndrome patients
}

\author{
Lisa Hinz ${ }^{1}$, Joan Torrella Barrufet ${ }^{1}$ and Vivi M. Heine ${ }^{1,2^{*}}$
}

\begin{abstract}
Rett Syndrome (RTT) is a neurodevelopmental disorder caused by mutations in the Methyl CpG binding protein 2 (MECP2) gene. Deficient $\mathrm{K}^{+}-\mathrm{Cl}^{-}$co-transporter 2 (KCC2) expression is suggested to play a key role in the neurodevelopmental delay in RTT patients' neuronal networks. KCC2 is a major player in neuronal maturation by supporting the GABAergic switch, through the regulation of neuronal chlorine homeostasis. Previous studies suggest that MeCP2 mutations lead to changed KCC2 expression levels, thereby causing a disturbance in excitation/ inhibition (E/I) balance. To investigate this, we performed protein and RNA expression analysis on post mortem brain tissue from RTT patients and healthy controls. We showed that KCC2 expression, in particular the KCC2a isoform, is relatively decreased in RTT patients. The expression of $\mathrm{Na}^{+}-\mathrm{K}^{+}-\mathrm{Cl}^{-}$co-transporter 1 (NKCC1), responsible for the inward transport of chlorine, is not affected, leading to a reduced KCC2/NKCC1 ratio in RTT brains. Our report confirms KCC2 expression alterations in RTT patients in human brain tissue, which is in line with other studies, suggesting affected $\mathrm{E} / \mathrm{l}$ balance could underlie neurodevelopmental defects in RTT patients.
\end{abstract}

Keywords: Rett syndrome, KCC2-deficiency, E/I imbalance, Neuronal network immaturity

\section{Introduction}

Rett syndrome (RTT) is a progressive neurodevelopmental disorder mainly affecting young girls. First clinical symptoms typically appear between 6 and 18 months of age, involving mental impairment, stereotypic behaviour, breathing abnormalities and severe seizures [3, 4, 18, 25]. Pathological studies showed that patients with RTT have a neuronal maturation defect with smaller soma, shorter dendrites, reduced synapses and reduced inhibition, leading to severe seizures $[4,18,25]$. Classical RTT is caused by various mutations within the Methyl-CpGbinding-protein 2 gene (MECP2), which protein has key functions in gene regulation and chromatin modulation. Because of its DNA-binding properties, lack of functional MeCP2 is affecting hundreds of target genes, complicating the insight into how MeCP2 deficiencies lead

\footnotetext{
* Correspondence: vm.heine@amsterdamumc.nl

'Department of Complex Trait Genetics, Center for Neurogenomics and Cognitive Research, Amsterdam Neuroscience, Vrije Universiteit, Amsterdam, The Netherlands

${ }^{2}$ Pediatric Neurology, Emma Children's Hospital, Amsterdam UMC, Vrije Universiteit Amsterdam, Amsterdam Neuroscience, Boelelaan 1085, 1081HV Amsterdam, The Netherlands
}

to brain abnormalities in RTT. One of the genes that is thought to play a critical role in delayed neuronal development in Rett patients, is the $\mathrm{K}^{+}-\mathrm{Cl}^{-}$co-transporter 2 (KCC2).

$\mathrm{KCC} 2$ is a chlorine transporter that regulates the intracellular chloride homeostasis in neurons together with the $\mathrm{Na}^{+}-\mathrm{K}^{+}-\mathrm{Cl}^{-}$co-transporter 1 (NKCC1) [6]. During early brain development, NKCC1 is the dominant transporter, thereby keeping the intracellular chloride concentration high. As gamma-aminobutyric acid (GABA) transmission is connected to the chloride gradient, GABA has excitatory functions in young neurons [8]. KCC2 exists in two isoforms, KCC2a and KCC2b [30]. During neuronal maturation, $\mathrm{KCC} 2 \mathrm{~b}$ levels in particular gradually increase, while $\mathrm{KCC} 2 \mathrm{a}$ levels only moderately change [31]. Increase in KCC2, results in lower intracellular chloride concentrations and a reversal of the chloride gradient. Consequently, the function of GABA in neurotransmission switches from excitation to inhibition $[7,9,13,19]$.

KCC2 level deficits are found in both rodent and human induced pluripotent stem cell models for RTT [5, 
$14,18,27]$. Interestingly, KCC2-deficient mice show similarities to Mecp2-knockout mice, including poor motor abilities, breathing abnormalities, impaired learning abilities and severe seizures [5, 17, 21, 23, 28, 29]. Based on excitatory neurotransmission, Kcc2-knockout mice display an immature neuronal phenotype and Mecp2-knockouts show a reduced GABAergic inhibition. In iPSC-based models, it has been shown that overexpression of $\mathrm{KCC} 2$ in iPSC-derived neurons from RTT patients can rescue GABAergic function [27], and it has been hypothesized that changes in neuronal development are linked to affected KCC2 levels.

In RTT patients, researchers have previously reported a decrease in KCC2 protein level in cerebrospinal fluid (CSF) samples of 2 to 29 year old female RTT patients and an altered $\mathrm{KCC} 2 / \mathrm{NKCC} 1$ ratio when compared to control samples [11]. More recently, these findings were confirmed on motor cortex and cerebellar tissue from RTT patients describing a reduction in $K C C 2$ expression levels [15]. However, the effect on KCC2 levels in other brain regions of RTT patients was not investigated. Here we investigated $K C C 2$ levels and $K C C 2 / N K C C 1$ ratio in post mortem tissue of patients and controls in the areas Brodmann Area (BA) 4, BA6, BA10, BA20 and Hippocampus, using RNA and immunocytochemical analysis. We found that $K C C 2$ expression is overall decreased in RTT patient post mortem tissue, suggesting playing a role in neuronal development. In particular, we identified $K C C 2 a$ but not $K C C 2 b$ expression being significantly decreased. Our findings, together with previous studies suggest, that alteration of KCC2 levels in RTT patients play a role in disease progression and support the hypothesis of ion channel gene dysfunction in RTT.

\section{Case report}

\section{Reduction of KCC2 immunofluorescence in post mortem} brain tissue from RTT patients

In this case report, we compared post mortem brain tissue from three female RTT patients with an average age of 20 years (\#UMB 4516, 20,98 years, p.R255X mutation in MECP2; \#UMB 4882, 17.85 years, p.R255X mutation in $M E C P 2$; \#UMB 5723, 22.5 years, no information about mutation available) with three gender and age matched controls (\#UMB 5602, 22.14 years; \#UMB 5646, 20.45 years; \#UMB 5670, 17.27 years). Samples from different BA were requested (BA4, BA6, BA10, BA20 and hippocampus) and kindly provided by the NICHD Brain and Tissue Bank for Developmental Disorders, University of Maryland, Baltimore, USA. Samples were shipped as deep-frozen tissue blocks and stored at $-80^{\circ} \mathrm{C}$ upon arrival.

First, we set out to confirm previous findings of reduced KCC2 levels in human CSF and mouse models, by performing immunocytochemistry for $\mathrm{KCC} 2$ and
MeCP2 on BA20 of the provided patient and control material. For this, $9 \mu \mathrm{m}$ sections from BA20 were prepared on pre-cooled glass slides and fixated with $100 \%$ acetone for $10 \mathrm{~min}$. Slides were blocked for $1 \mathrm{~h}$ and incubated with antibodies (KCC2, Neuromab, 1:500; MeCP2, Cell Signalling, 1:500) over night at $4{ }^{\circ} \mathrm{C}$. Next day, sections were incubated with secondary antibodies (AlexaFluor anti rabbit 594, 1:1000, Thermo Fisher Scientific; Alexa-Fluor anti mouse 488, 1:1000, Thermo Fisher Scientific) for $1 \mathrm{~h}$ at RT, followed by DAPI treatment for 5 min. Slides were mounted with Fluoromount G (Thermo Fisher Scientific) and imaged by fluorescent microscopy (Leica). Pictures were transferred to Java-based imaging software Fiji (Analysis Find Maxima). We averaged data derived from 2 images per patient or healthy control.

Sections from healthy controls presented positive MeCP2 staining in all cells (Fig. 1a), while RTT patient tissue showed a mosaicism of positive and negative nuclei (Fig. 1b). KCC2 staining was located along the dendrites and soma of neurons (Fig. 1a+b). Although, KCC2 expression levels by fluorescent intensity measurements did not reach statistical significance (Mann Whitney Test, $p=0.1$ ), it was decreased in patients when compared to healthy controls (Fig. $1 \mathrm{~b}+\mathrm{c}$ ).

\section{Alteration of $K C C 2$ RNA expression level in post mortem brain tissue of Rett syndrome patients}

To further investigate if gene expression level of $K C C 2$ is altered in samples of RTT patients' brain tissue, we analysed RNA levels by quantitative real time polymerase chain reaction (qPCR). Therefore, RNA was collected from frozen post mortem brain tissue, using standardized trizol/chloroform-RNA isolation followed by reverse transcriptase PCR with SuperScript IV Reverse Transcriptase (Thermo Fisher Scientific). For qPCR analysis, SYBR Green Real-Time PCR Master Mix was used with $100 \mathrm{ng}$ of sample cDNA as well as forward and reverse primer of required gene of interest (Table 1). qPCR analysis was performed with Roche Light Cycler 480 and results were analysed by using Roche Light Cycler 480 Software.

KCC2 levels in the RTT brain were determined by averaging the measured levels in five brain regions, BA4, BA6, BA10, BA20 and hippocampus, relative to housekeeping genes. We found that all three RTT patients studied, show a lower KCC2 expression compared to healthy controls (Kruskal-Wallis test $p=0,0143$, Fig. 2a). When brain regions were studied separately, KCC2 expression levels were also decreased in patients, but only reached statistical significance in BA6 (one-way ANOVA, BA6 $p<0.004$, BA4 n.s., BA10 n.s., BA20 n.s., Hippocampus n.s.) (Fig. 2b). No alterations were observed in NKCC1 expression levels of RTT patients when compared to controls (Fig. 2c). Therefore, mean 

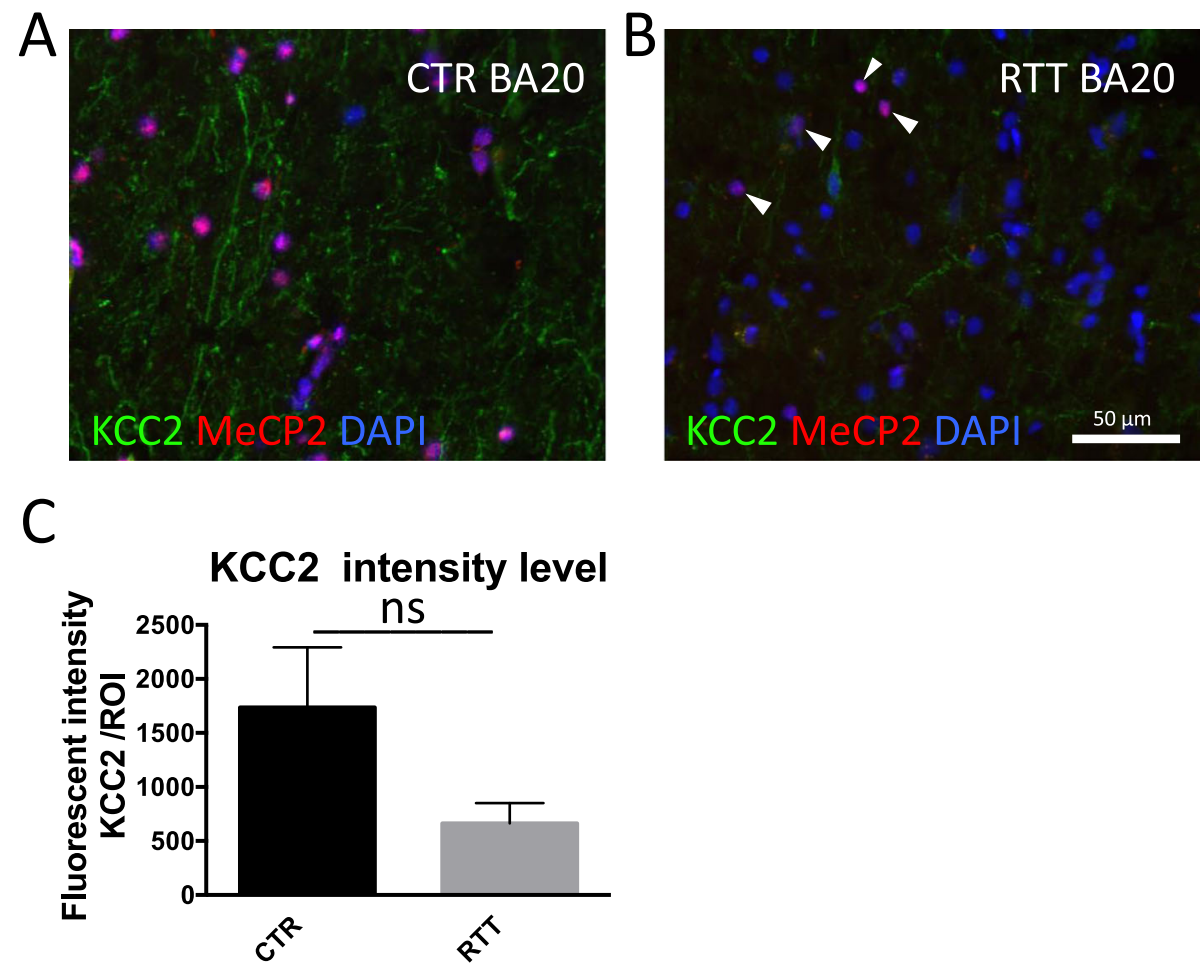

Fig. 1 KCC2 immunohistochemistry in RTT patient brain. Representative image of immunofluorescent staining for KCC2 and MeCP2 in BA 20 of control (a) and RTT patient (b) tissue. Because of mosaic expression, MeCP2-positive cells are also detected in RTT patient tissue marked by arrowheads (b). Fluorescent intensity measurements indicated reduced KCC2 levels in RTT patient (Mann Whitney Test, ns.) (c)

ratio of $K C C 2$ and $N K C C 1$ of all three patients were significantly decreased (Mann-Whitney test, $p<0.007$ ) (Fig. $2 \mathrm{~d})$. We also looked into the expression levels of the different subtypes of $K C C 2$, namely $K C C 2 a$ and $K C C 2 b$. Both isoform were decreased in RTT patient samples (average of the different brain regions), but only reached statistical significance for $K C C 2 a$ (Fig. 2e). Therefore, our results are in line with previous findings, that $K C C 2$ expression is affected by RTT.

Table 1 Primers qPCR

\begin{tabular}{lll}
\hline 1 & RPL13 forward & GAGACAGTTCTGCTGAAGAACTGAA \\
2 & RPL13 reverse & TCCGGACGGGCATGAC \\
3 & GAPDH forward & TCAAGGGCATCCTGGGCTAC \\
4 & GAPDH reverse & CGTCAAAGGTGGAGGAGTGG \\
5 & KCC2 forward & ACATCTTGGCGTCATCCTC \\
6 & KCC2 reverse & CAGGCACAACACCATTCGTT \\
7 & NKCC1 forward & CCGATTTCGAGAGGAAGAG \\
8 & NKCC1 reverse & TGCAATTCCTACGTAAACCAA \\
9 & KCC2 $a$ forward & AGAAGCCCTGACCCAGAGTC \\
10 & KCC2a reverse & CTTCTCTGTGTCGGTGCTGT \\
11 & KCC2b forward & CGCCACCATGCTAAACAACC \\
12 & KCC2b reverse & CTTCTCTGTGTCGGTGTGT \\
\hline
\end{tabular}

\section{Discussion}

Earlier studies investigated KCC2 level changes in RTT patient's CSF, in human iPSC-derived neurons and in motor cortex and cerebellar tissue of RTT patients [11, $15,27]$. These findings suggest involvement of KCC2 in disease mechanisms in RTT. In support of this hypothesis and to study the influence of MeCP2 on KCC2 levels in different brain areas, we investigated $K C C 2$ expression in four different cortical areas and hippocampus of three RTT patients and age matched controls. We observed a significant reduction in $K C C 2$ gene expression in RTT samples compared to controls. Considering $\mathrm{KCC} 2$ is essential for a functional E/I balance [6, 7], these insights strengthen the importance of ion channel dysfunction in RTT patient brains, as E/I imbalance are associated with delayed neuronal development and seizures in RTT.

RTT tissue was derived from three female patients. Due to random X-chromosomal inactivation during development, the derived tissue showed mosaicism for MECP2 expression [24]. Therefore, the observed BAs did contain mixed populations of affected and unaffected neurons (Fig.1b). If we assume that $\mathrm{MeCP} 2$ deficiencies lead to reduced $\mathrm{KCC} 2$ levels, it is likely that $\mathrm{KCC} 2$ levels were not affected in all cells of the studied tissue. This could explain the observed decrease in fluorescent 


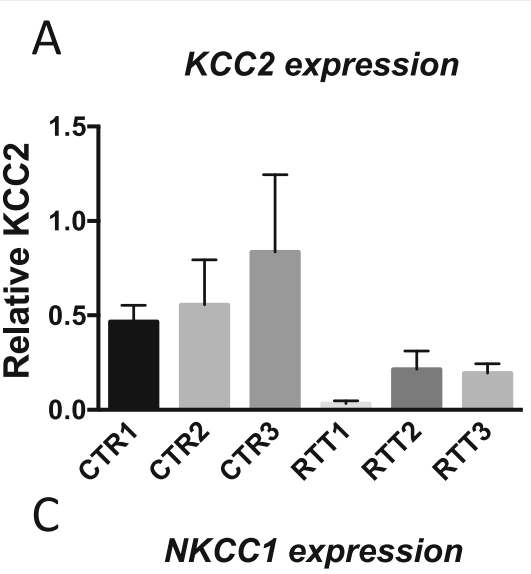

\section{B KCC2 expression}
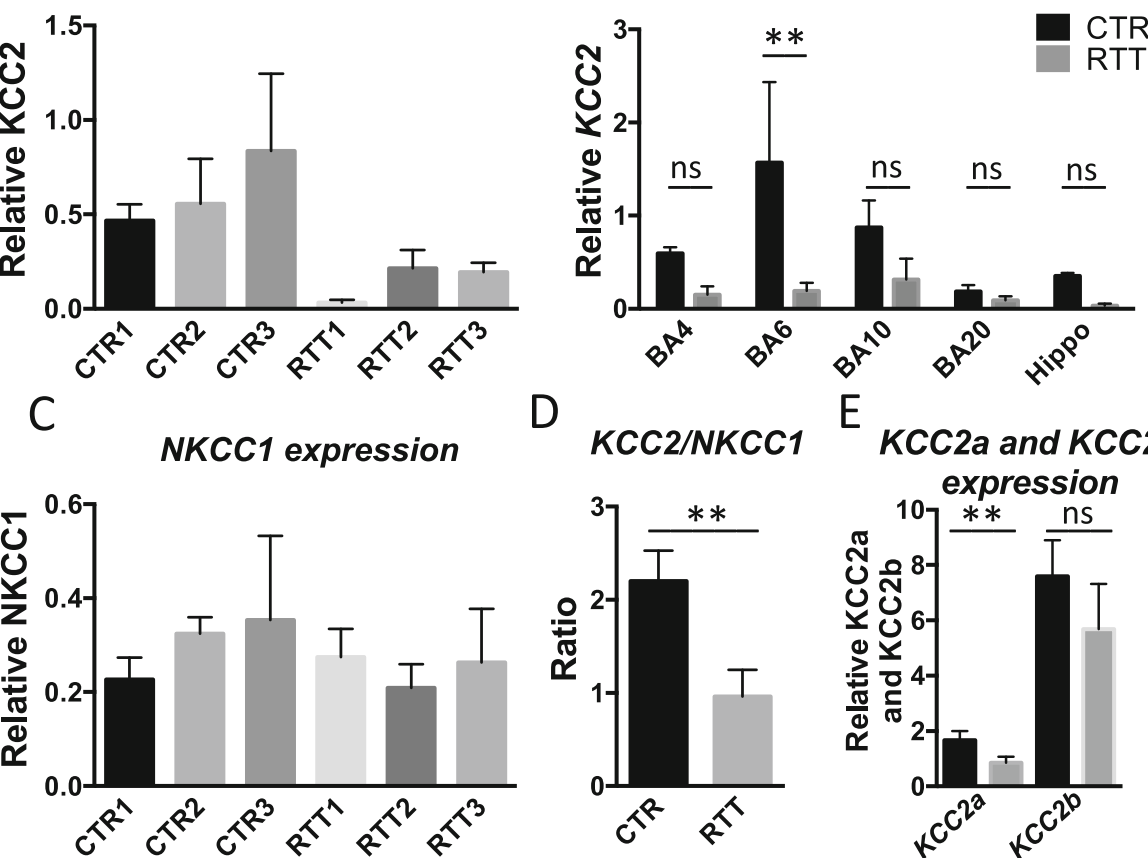

D
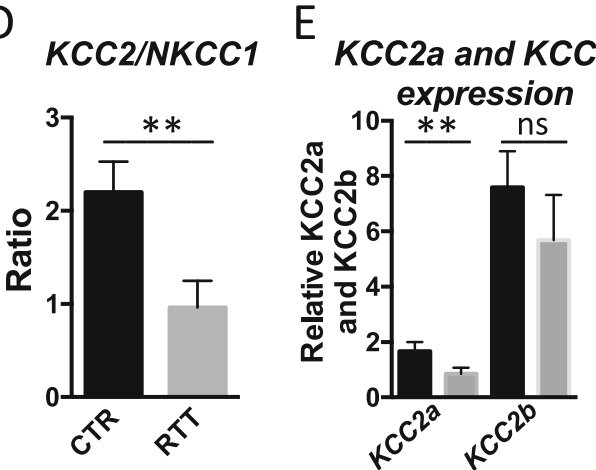

Fig. 2 qPCR analysis for KCC2 and NKCC1 in RTT brain samples. KCC2 expression levels are reduced in all 3 RTT patient brains compared to control 3 control brains (a). Reduced KCC2 expression levels are found in all brain regions studied, but only reached statistical significance in BA6 (Multiple t test BA6 $p<0.01$; one-way ANOVA $P<0,0001)$ (b). NKCC1 expression levels are unchanged in RTT patient brains (c). The KCC2/NKCC1 ratio is significantly reduced in RTT patient brains (Mann-Whitney test $P<0,007)(\mathbf{d})$. Averaged expression levels of $K C C 2$ isotypes are reduced in RTT samples, which reached statistical significance for KCC2a (2-Way-ANOVA $P<0,005)$ but not for KCC2b expression (2-Way-ANOVA, ns) (e)

intensity of KCC2 immunostainings in RTT brain tissue without reaching statistical significance (Fig. 1c). Studying MeCP2-negative cells exclusively, could overcome this issue. But, due to pre-treatment of the brain tissue, namely freezing and cryo-sectioning, identification of individual cells was difficult. Therefore, only the average fluorescent intensity of KCC2 could be analysed.

To gain more insight into $K C C 2$ expression levels within RTT patient brains, we additionally performed qPCR analysis. We showed decreased $K C C 2$ expression in different areas of RTT patient brains compared to healthy controls (Fig. 2b). Noteworthy, we observed that KCC2 expression was not evenly expressed over control tissue, which could reflect expression changes during developmental stages of the brain areas [22] and regionspecific differences [16]. But, as controls were agematched, these variables do not account for decreased KCC2 levels observed. Also here mosaic expression could have played a role. However, we did observe a similar trend for all brain areas investigated, showing a reduction of $K C C 2$ expression in RTT compared to CTR samples. These results are in line with the immunofluorescent intensity analysis of BA20, suggesting that protein levels of $\mathrm{KCC} 2$ are reduced in several different brain areas in the cortex and hippocampus.
Besides $K C C 2$, we also investigated $N K C C 1$ expression levels and did not detect expression level alterations. These findings are in line with previous studies in human CSF and brain tissue [11, 15]. However, as the KCC2/NKCC1 ratio ultimately regulates chloride homeostasis, GABA switch and maturation of the brain, changed KCC2 levels can have a major impact on brain development $[6,7,20]$. As brain tissue samples were taken from RTT patients and healthy controls in adolescence, we cannot exclude KCC2 levels normalize over time. However, by the end of adolescence, developmental processes of the brain are almost completed and KCC2 levels will most likely remain stable. Therefore, it is likely that alterations in $K C C 2$ expression already appear during early brain development, having a major impact on disease progression in RTT patients.

Interestingly, we identified a significant reduction in $K C C 2 a$ but not in $K C C 2 b$ expression levels. As $K C C 2 b$ expression is strongly upregulated during early development, it is suggested that $\mathrm{KCC} 2 \mathrm{~b}$ is primarily responsible for the GABAergic shift [30]. However, before KCC2b levels majorly increase, KCC2a levels make up $50 \%$ of total KCC2 levels. These expression levels double in cortical areas during development, suggesting a significant role in network maturation [31]. Nonetheless, in 
adolescence only $8-10 \%$ of the total $\mathrm{KCC} 2$ levels involves KCC2a [30, 31]. Worth mentioning, an important role for KCC2a in development of respiratory rhythmogenesis has been shown, as its absence leads to abnormal breathing rates and apnoea in mice [12]. Breathing abnormalities with apnoea are common symptoms in RTT patients and are so far associated with reduced GABAergic innervation in the brain stem $[1,10,26]$. Since $K C C 2 a$ is specifically highly expressed within the brain stem, the role of KCC2a level changes deserves further study.

Summarizing, our results confirm earlier hypotheses that RTT patient brains show a decreased $K C C 2$ expression, resulting in a lower $K C C 2 / N K C C 1$ ratio. Interestingly, we could identify significant changes in the expression of $\mathrm{KCC} 2 a$ isoform. Therefore, brains of RTT patients display an underdeveloped phenotype, which matches other phenotypic alterations, such as decreased neuronal size, reduced number of synapses and shorter dendritic outgrowth $[2-4,18,25]$. This proposes the need for sufficient treatment during these developmental processes to support brain development and reduce disease progression. By understanding these mechanisms and its alterations in RTT during early development, we can identify new treatment approaches and elucidate the need for early diagnostics.

\section{Abbreviations}

BA: Brodmann Area; CSF: Cerebral Spinal Fluid; E/l: Excitation/Inhibition; GABA: Gamma-Aminobutyric Acid; KCC2: $\mathrm{K}^{+}-\mathrm{Cl}^{-}$Co-Transporter 2; MeCP2: Methyl-CpG-Binding-Protein 2; NKCC1: $\mathrm{Na}^{+}-\mathrm{K}^{+}-\mathrm{Cl}^{-} \mathrm{Co}-$ Transporter 1; qPCR: Quantitative Real Time Polymerase Chain Reaction; RTT: Rett Syndrome

\section{Acknowledgements}

Human brain tissue was kindly provided by NICHD, Brain and Tissue Bank for Developmental Disorders, University of Maryland, Baltimore, USA. We thank the European Commission and the EU IDP Project CognitionNet for funding.

\section{Authors' contributions}

$\mathrm{LH}$ analyzed and interpreted the immunofluorescent staining and $\mathrm{QPCR}$ data data and wrote the first draft of the manuscript. JTB performed the histological examination of the brain tissue. VH supervised planning of experiments and writing of the manuscript. All authors read and approved the final manuscript.

\section{Funding}

This work was funded by the EC under FP7-PEOPLE-2013 (607508).

\section{Availability of data and materials}

Not applicable.

\section{Ethics approval and consent to participate}

We thank the University of Maryland Brain and Tissue Bank in Baltimore for providing us human post mortem brain samples.

\section{Consent for publication}

Not applicable.

\section{Competing interests}

The authors declare that they have no competing interests.
Received: 30 September 2019 Accepted: 21 November 2019

Published online: 03 December 2019

\section{References}

1. Abdala AP, Toward MA, Dutschmann M, Bissonnette JM, Paton JFR (2016) Deficiency of GABAergic synaptic inhibition in the Kölliker-Fuse area underlies respiratory dysrhythmia in a mouse model of Rett syndrome. J Physiol 594:223-237

2. Armstrong D, Dunn JK, Antalffy B, Trivedi R (1995) Selective dendritic alterations in the cortex of Rett syndrome. J Neuropathol Exp Neurol 54: 195-201

3. Armstrong DD (2001) Rett syndrome neuropathology review 2000. Brain and Development 23:72-76

4. Armstrong DD (2005) Neuropathology of Rett syndrome. J Child Neurol 1: 259-288

5. Banerjee A, Rikhye RV, Breton-Provencher V, Tang X, Li C, Li K et al (2016) Jointly reduced inhibition and excitation underlies circuit-wide changes in cortical processing in Rett syndrome. Proc Natl Acad Sci U S A 113:E7287E7296

6. Ben-Ari Y (2002) Excitatory actions of gaba during development: the nature of the nurture. Nat Rev Neurosci 3:728-739

7. Ben-Ari Y (2014) The GABA excitatory/inhibitory developmental sequence: a personal journey. Neuroscience 279:187-219

8. Ben-Ari Y, Gaiarsa J-L, Tyzio R, Khazipov R (2007) GABA: a Pioneer transmitter that excites immature neurons and generates primitive oscillations. Physiol Rev 87:1215-1284

9. Chamma I, Chevy Q, Poncer JC, Lévi S (2012) Role of the neuronal K-Cl cotransporter KCC2 in inhibitory and excitatory neurotransmission. Front Cell Neurosci 6:5

10. d'Orsi G, Demaio V, Scarpelli F, Calvario T, Minervini MG (2009) Central sleep apnoea in Rett syndrome. Neurol Sci 30:389-391

11. Duarte ST, Armstrong J, Roche A, Ortez C, Pérez A, O'Callaghan Mdel M et al (2013) Abnormal expression of cerebrospinal fluid Cation chloride Cotransporters in patients with Rett syndrome. PLoS One 8:1-7

12. Dubois CJ, Cardoit L, Schwarz V, Markkanen M, Airaksinen MS, Uvarov P et al (2018) Role of the K-Cl-Cotransporter KCC2a Isoform in Mammalian Respiration at Birth Significance Statement. ENeuro 5:264-282

13. Egorov AV, Draguhn A (2013) Development of coherent neuronal activity patterns in mammalian cortical networks: common principles and local hetereogeneity. Mech Dev 130:412-423

14. El-Khoury R, Panayotis N, Matagne V, Ghata A, Villard L, Roux JC (2014) GABA and glutamate pathways are spatially and developmentally affected in the brain of Mecp2-deficient mice. PLoS One 9:e92169

15. Gogliotti RG, Fisher NM, Stansley BJ, Jones CK, Lindsley CW, Conn PJ et al (2018) Total RNA sequencing of Rett syndrome autopsy samples identifies the M4 muscarinic receptor as a novel therapeutic target. J Pharmacol Exp Ther 365:291-300

16. Hawrylycz MJ, Lein ES, Guillozet-Bongaarts AL, Shen EH, Ng L, Miller JA et al (2012) An anatomically comprehensive atlas of the adult human brain transcriptome. Nature. 489:391-399

17. Hübner CA, Stein V, Hermans-Borgmeyer I, Meyer T, Ballanyi K, Jentsch TJ (2001) Disruption of KCC2 reveals an essential role of K-cl cotransport already in early synaptic inhibition. Neuron 30:515-524

18. Ip JPK, Mellios N, Sur M (2018) Rett syndrome: insights into genetic, molecular and circuit mechanisms. Nat Rev Neurosci 19:368-382

19. Ito S (2016) GABA and glycine in the developing brain. J Physiol Sci 66:375379

20. Kaila K, Price TJ, Payne JA, Puskarjov M, Voipio J (2014) Cation-chloride cotransporters in neuronal development, plasticity and disease. Nat Rev Neurosci 15:637-654

21. Kelley MR, Cardarelli RA, Smalley JL, Ollerhead TA, Andrew PM, Brandon NJ et al (2018) Locally reducing KCC2 activity in the Hippocampus is Suf fi cient to induce temporal lobe epilepsy. EBioMedicine 32:62-71

22. Kovács K, Basu K, Rouiller I, Sík A (2014) Regional differences in the expression of $\mathrm{K}+-\mathrm{Cl}-2$ cotransporter in the developing rat cortex. Brain Struct Funct 219:527-538

23. Pellegrino C, Gubkina O, Schaefer M, Becq H, Ludwig A, Mukhtarov M et a (2011) Knocking down of the KCC2 in rat hippocampal neurons increases intracellular chloride concentration and compromises neuronal survival. J Physiol 589:2475-2496 
24. Renthal W, Boxer LD, Hrvatin S, Li E, Silberfeld A, Nagy MA et al (2018) Characterization of human mosaic Rett syndrome brain tissue by singlenucleus RNA sequencing. Nat Neurosci 21:1670-1679

25. Smrt RD, Eaves-Egenes J, Barkho BZ, Santistevan NJ, Zhao C, Aimone JB et al (2007) Mecp2 deficiency leads to delayed maturation and altered gene expression in hippocampal neurons. Neurobiol Dis 27:77-89

26. Southall DP, Kerr AM, Tirosh E, Amos P, Lang MH, Stephenson JB (1988) Hyperventilation in the awake state: potentially treatable component of Rett syndrome. Arch Dis Child 63:1039-1048

27. Tang X, Kim J, Zhou L, Wengert E, Zhang L, Wu Z et al (2016) KCC2 rescues functional deficits in human neurons derived from patients with Rett syndrome. Proc Natl Acad Sci U S A 113:751-756 1524013113-

28. Tornberg J, Segerstråle M, Kulesskaya N, Voikar V, Taira T, Airaksinen MS (2007) KCC2-deficient mice show reduced sensitivity to diazepam, but Normal alcohol-induced motor impairment, Gaboxadol-induced sedation, and Neurosteroid-induced hypnosis. Neuropsychopharmacology 32:911-918

29. Tornberg J, Voikar V, Savilahti H, Rauvala H, Airaksinen MS (2005) Behavioural phenotypes of hypomorphic KCC2-deficient mice. Eur J Neurosci 21:1327-1337

30. Uvarov P, Ludwig A, Markkanen M, Pruunsild P, Kaila K, Delpire E et al (2007) A novel N-terminal isoform of the neuron-specific K-cl cotransporter KCC2. J Biol Chem 282:30570-30576

31. Uvarov P, Ludwig A, Markkanen M, Soni S, Hübner CA, Rivera C et al (2009) Coexpression and heteromerization of two neuronal K-cl cotransporter isoforms in neonatal brain. J Biol Chem 284:13696-13704

\section{Publisher's Note}

Springer Nature remains neutral with regard to jurisdictional claims in published maps and institutional affiliations.

Ready to submit your research? Choose BMC and benefit from:

- fast, convenient online submission

- thorough peer review by experienced researchers in your field

- rapid publication on acceptance

- support for research data, including large and complex data types

- gold Open Access which fosters wider collaboration and increased citations

- maximum visibility for your research: over $100 \mathrm{M}$ website views per year

At $\mathrm{BMC}$, research is always in progress.

Learn more biomedcentral.com/submissions 